\title{
Cor do solo na identificação de áreas com diferentes potenciais produtivos e qualidade de café
}

\author{
Danilo Almeida Baldo do Carmo(1), José Marques Júnior ${ }^{(1)}$, Diego Silva Siqueira(1), \\ Angélica Santos Rabelo de Souza Bahia ${ }^{(1)}$, Henrique Menezes Santos ${ }^{(1)}$ e Gustavo Zanetti Pollo(2)
}

\begin{abstract}
(1)Universidade Estadual Paulista, Faculdade de Ciências Agrárias e Veterinárias, Departamento de Solos e Adubos, Via de Acesso Prof. Paulo Donato Castellane, s/no, CEP 14884-900 Jaboticabal, SP, Brasil. E-mail: danilo_baldo@hotmail.com, marques@fcav.unesp.br, diego_silvasiqueira@yahoo.com.br, angelicasantosrabelo@yahoo.com.br, henrique-menezes@hotmail.com (2)Grupo Cambuhy Agrícola, Rodovia Whashington Luiz, Km 307.3, CEP 5995-900 Matão, SP, Brasil. E-mail: guzpollo@yahoo.com.br
\end{abstract}

Resumo - O objetivo deste trabalho foi verificar a eficácia da cor do solo na identificação de áreas com diferentes potenciais produtivos e qualidade de café, em Argissolo Vermelho-Amarelo desenvolvido de arenito, no Planalto Ocidental Paulista, no Estado de São Paulo. Coletaram-se 173 amostras em 39 ha, para a determinação de matiz, valor, croma e índice de avermelhamento do solo, com base nos dados da espectroscopia de reflectância difusa (ERD). Avaliaram-se, também, a produtividade e a qualidade do fruto de café, e calculou-se o índice de diagnose foliar (DRIS). A maior correlação espacial observada pelo variograma cruzado ocorreu entre matiz e DRIS, em pontos distantes em até $497 \mathrm{~m}$. A menor correlação espacial foi observada em croma e produção, em pontos distantes em até $207 \mathrm{~m}$. As áreas com o maior potencial de produtividade ( 20 a 33 sacas por hectare) apresentaram matiz entre 6,99 e 7,06, croma entre 5,00 e 5,08 e valor entre 4,40 e 4,45. As áreas com o menor potencial de produtividade ( 3 a 7 sacas por hectare) apresentaram matiz entre 7,27 e 7,68, croma entre 5,20 e 5,28 e valor entre 4,51 e 4,67. Nestes locais, o teor de argila foi de $16 \mathrm{~g} \mathrm{~kg}^{-1}$, os teores de $\mathrm{P}$ variaram entre $65 \mathrm{e}$ $75 \mathrm{mg} \mathrm{dm}^{-3}$, e a soma de bases estava entre 56 e $58 \mathrm{mmol}_{\mathrm{c}} \mathrm{dm}^{-3}$. O uso da cor determinada por ERD é eficaz para identificar áreas com diferentes potenciais produtivos e qualidade de café, com precisão de 61 a 97\%.

Termos para indexação: Coffea arabica, cor do solo, geoestatística, pedometria.

\section{Soil color for the identification of areas with different yield potential and coffee quality}

\begin{abstract}
The objective of this work was to verify soil color effectiveness for the identification of areas with different yield potentials and coffee quality, in an Ultisol developed from sandstone, in Planalto Ocidental Paulista, in the state of São Paulo, Brazil. One hundred seventy-three samples were collected from 39 ha, for the determination of hue, value, chroma, and the soil redness index, based on data of diffuse reflectance spectroscopy (DRS). Productivity and quality of coffee fruit were also evaluated, and the leaf diagnosis index (DRIS) was calculated. The highest spatial correlation, observed in the cross variogram, occurred between hue and DRIS in points up to $497 \mathrm{~m}$ apart. The lowest spatial correlation was observed for chroma and production, in points spaced by up to $207 \mathrm{~m}$. The areas with the highest yield potential ( 20 to 33 bags per hectare) showed hue between 6.99 and 7.06, croma between 5.00 and 5.08, and value between 4.40 and 4.45 . The areas with the lowest productivity potential ( 3 to 7 bags per hectare) showed hue between 7.27 and 7.68, chroma between 5.20 and 5.28, and value between 4.51 and 4.67. In these places, clay content was of $16 \mathrm{~g} \mathrm{~kg}^{-1}, \mathrm{P}$ levels varied between 65 and $75 \mathrm{mg} \mathrm{dm}^{-3}$, and the sum of bases was between 56 and $58 \mathrm{mmol}_{\mathrm{c}} \mathrm{dm}^{-3}$. The use of the color determined by DRS is effective to identify areas with different productive potentials and coffee quality, with 61 to $97 \%$ accuracy.
\end{abstract}

Index terms: Coffea arabica, soil color, geostatistics, pedometrics.

\section{Introdução}

O café é classificado com base em parâmetros qualitativos, como defeitos no grão e qualidade da bebida. A variabilidade natural do solo e de seus atributos, em nível detalhado ( $<1: 5.000)$, é uma das causas da variação quantitativa e qualitativa da resposta do café, expressa em diferentes produtividades e qualidade de grãos. Entre as dificuldades de elaboração de modelos de previsão para a cultura do café está a caracterização da variabilidade natural dos solos e seus atributos (Sanchez et al., 2013).

$\mathrm{Na}$ mesma área, podem ser encontrados cultivos com baixa produtividade e qualidade de grão e que, 
no momento da colheita, são agrupados aos de alta produção e qualidade (Sanchez et al., 2013). Essa mistura pode provocar uma diminuição do rendimento final de até 2,5 vezes, considerando-se o ganho das sacas beneficiadas e o custo com adubação (Pollo, 2013). Uma alternativa para auxiliar o planejamento estratégico e a tomada de decisão do cafeicultor pode ser a definição de áreas de manejo específico, que apresentem máxima homogeneidade dos atributos do solo.

Ferramentas computacionais - como o modelo digital de elevação (MDE), a geoestatística, a análise multivariada e a integração destas ferramentas - têm sido cada vez mais utilizadas em ciências agrárias para auxiliar a identificação de áreas homogêneas (Siqueira et al., 2010; Sanchez et al., 2013).

A evolução computacional e a formação de pessoas para utilizar essas ferramentas no planejamento agrícola ocorre em paralelo a outras análises de campo e laboratório, que têm sido testadas para a identificação de áreas de manejo específico, entre as quais a quantificação da cor do solo por espectroscopia de refletância difusa (ERD) (Marques Jr. et al., 2014). Com à evolução dos sensores e de técnicas mais refinadas de quantificação, a cor ganha novas aplicações e perspectivas para a caracterização da variabilidade dos atributos do solo de caráter agronômico, como o teor de argila, o fósforo adsorvido e a saturação por bases, por exemplo (Marques Jr. et al., 2014; Camargo et al., 2015). Os parâmetros da cor do solo (matiz, valor, croma), quando determinados pela leitura da reflectância do solo na faixa do visível (350 a 750 $\mathrm{nm}$ ), podem ser utilizados na estimativa do teor de óxidos de ferro, como hematita e goethita (Viscarra Rossel et al., 2010; Bahia et al., 2015), carbono total (Xie et al., 2011) e outras importantes propriedades do solo que covariam com ela, tanto do ponto de vista pedológico quanto do agronômico (Barrón et al., 2000; Barrón \& Torrent, 2013). A cor pode ser determinada de diferentes maneiras (Torrent \& Barrón, 2008). Em ciências agrárias, é comum utilizar-se a comparação visual de padrões de cor conforme a caderneta de Munsell (Soil Color Charts) que, no entanto, apresenta alguma dificuldade de comparação dos resultados, em razão das diferenças de percepção visual entre os avaliadores (Barrón et al., 2000; Campos \& Demattê, 2004). Apesar de o sistema Munsell ter sido proposto inicialmente em ciências agrárias, para fins pedológicos na classificação de solos, ele pode ser utilizado para a definição de áreas de manejo específico para as culturas da cana-de-açúcar (Marques Jr. et al., 2014) e da soja (Resende et al., 2014), estudos de gênese de solo (Dantas et al., 2014a), quantificação do potencial de erosão (Dantas et al., 2014a), e identificação de áreas com diferentes potenciais de adsorção de fósforo (Camargo et al., 2015; Peluco et al., 2015).

Nesse contexto, o emprego da ERD para a determinação dos parâmetros da cor tem-se mostrado útil para a caracterização quantitativa e mais precisa dos solos e mostrado que é possível explorar melhor esta propriedade a partir de um instrumento não muito complexo, além de promover o desenvolvimento científico e aplicações neste campo de estudo em ciência do solo (Barrón et al., 2000; Torrent \& Barrón, 2008; Barrón \& Torrent, 2013).

O objetivo deste trabalho foi verificar a cor de solo, quantificada por espectroscopia de reflectância difusa (ERD), para a identificação de áreas com diferentes potenciais produtivos e qualidade de café, em um Argissolo Vermelho-Amarelo desenvolvido de arenito, no Planalto Ocidental Paulista.

\section{Material e Métodos}

O trabalho foi realizado em uma área de 39 ha, no Município de Matão, no sudoeste do Estado de São Paulo (Figura 1) - a $21^{\circ} 36^{\prime} \mathrm{S}$ e $48^{\circ} 29^{\prime} \mathrm{W}$, com altitude entre 590 e $615 \mathrm{~m}$. Segundo a classificação de Thornthwaite (1948), o clima local é definido como B1rB'4a', tipo mesotérmico úmido, com pequena deficiência hídrica, com evapotranspiração de verão abaixo de $70 \%$ da evapotranspiração anual.

O material geológico está relacionado aos arenitos do Grupo Bauru. Geomorfologicamente, a área está inserida no Planalto Ocidental Paulista, que ocupa aproximadamente metade da extensão territorial do Estado de São Paulo - cerca de 13 milhões de hectares (IPT, 1981).

O solo da área de estudo foi classificado como Argissolo Vermelho-Amarelo distrófico (Santos et al., 2006), com teor médio de $\mathrm{Fe}_{2} \mathrm{O}_{3}$ (obtido por ataque sulfúrico) de $30 \mathrm{~g} \mathrm{~kg}^{-1}$. À profundidade de 0,0-0,2 $\mathrm{m}$, o teor médio de argila é de $160 \mathrm{~g} \mathrm{~kg}^{-1}$, a soma de bases é de $52 \mathrm{mmol}_{\mathrm{c}} \mathrm{dm}^{-3}$, e a suscetibilidade magnética é de $1,5010^{-6} \mathrm{~m}^{3} \mathrm{~kg}^{-1}$. Realizou-se, também, a avaliação da espessura dos horizontes $\mathrm{A}+\mathrm{E}$, por 
meio de três tradagens profundas sob a projeção da copa da planta central da unidade amostral (Figura 1 A) e, posteriormente, calculou-se a média das determinações. Estes valores foram utilizados na modelagem do variograma e elaboração do mapa de espessura do horizonte $\mathrm{A}+\mathrm{E}$, para complementar a caracterização da área e auxiliar a compreensão das relações de causa e efeito entre solo e paisagem. A mudança textural em decorrência do maior teor de argila, na camada mais profunda, foi utilizada como parâmetro indicativo da profundidade dos horizontes A $+E$, conforme realizado por Sanchez et al. (2009, 2013).

A área é cultivada há 25 anos com Coffea arabica 'Catuaí Amarelo IAC 62'. À época do estudo, as plantas estavam com 7 anos de idade, dispostas em espaçamento $3,5 \times 0,5 \mathrm{~m}$, cultivadas desde o início em sistema de fertirrigação por gotejamento. Coletaramse amostras de solo à profundidade de $0,0-0,20 \mathrm{~m}$, a intervalos regulares de $45 \mathrm{~m}$, sob a projeção da copa da planta central, num total de 173 pontos de amostragem. Para a avaliação da produtividade e da qualidade do fruto, definiram-se unidades amostrais de nove plantas, tendo-se avaliado o total de 1.557 plantas.

As amostras de solo coletadas na malha e nas trincheiras foram submetidas à análise granulométrica, em solução de $\mathrm{NaOH}$ 0,1 N como dispersante químico, com agitação em aparato de baixa rotação (Day, 1965). A argila foi determinada pelo método da pipeta. Cálcio,

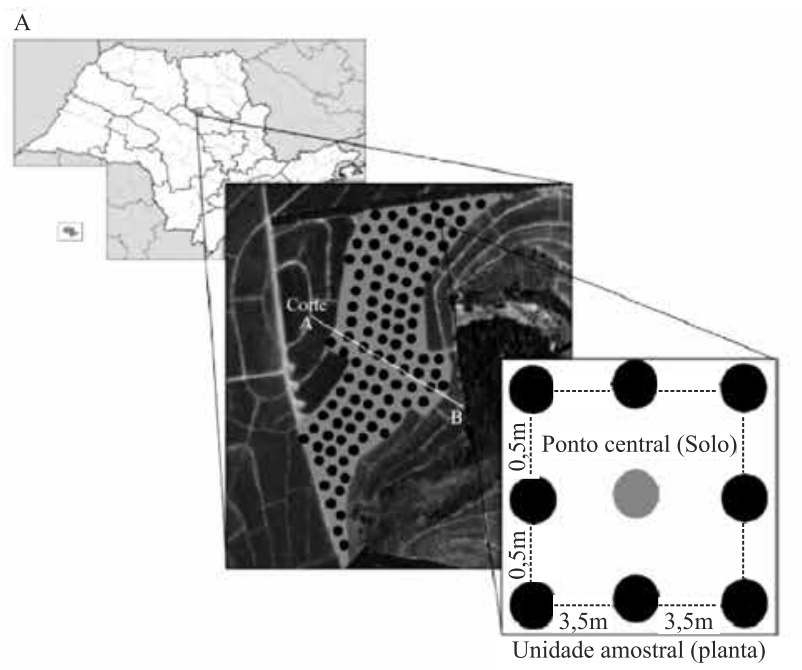

magnésio e potássio trocáveis e o fósforo disponível foram extraídos pelo método da resina trocadora de íons (Raij et al., 1987), e o carbono orgânico foi extraído conforme Claessen (1997).

Para a obtenção dos espectros de reflectância difusa utilizou-se o sensor Lambda 950 UV/VIS/NIR. Usouse aproximadamente $0,5 \mathrm{~g}$ de terra fina seca ao ar (TFSA), moída em almofariz de ágata até a obtenção de coloração constante. Os valores de reflectância foram determinados a cada $0,5 \mathrm{~nm}$ na faixa do visível, no intervalo de 380 a $780 \mathrm{~nm}$ (Torrent \& Barrón, 2008).

Após a obtenção dos espectros de reflectância das amostras de solo, determinaram-se os valores triestímulo XYZ, definidos pela Comisión Internacional de L'Eclairage - CIE (Wyszecki \& Stiles, 1982) e, a partir destas coordenadas, foram deduzidos os valores de Munsell referentes a matiz $(\mathrm{H})$, croma (C) e valor (V), pelo programa Munsell Conversion, versão 6.4. Os valores de $\mathrm{H}, \mathrm{C}$ e $\mathrm{V}$, conforme método de Munsell, foram utilizados no cálculo do índice de avermelhamento (IAV), pela equação 1 (Torrent \& Barrón, 2008), a seguir: IAV $=(10-\mathrm{H}) \times \mathrm{C} / \mathrm{V}$, em que: $\mathrm{V}$ é o valor Munsell; $\mathrm{C}$ é o valor numérico do croma; $\mathrm{e}$ $\mathrm{H}$ é o valor numérico que corresponde ao matiz.

A produtividade foi estimada com base na produção das plantas da unidade amostral. A classificação do tamanho dos grãos foi realizada por um jogo de peneiras que os separa pelo tamanho e pela forma.
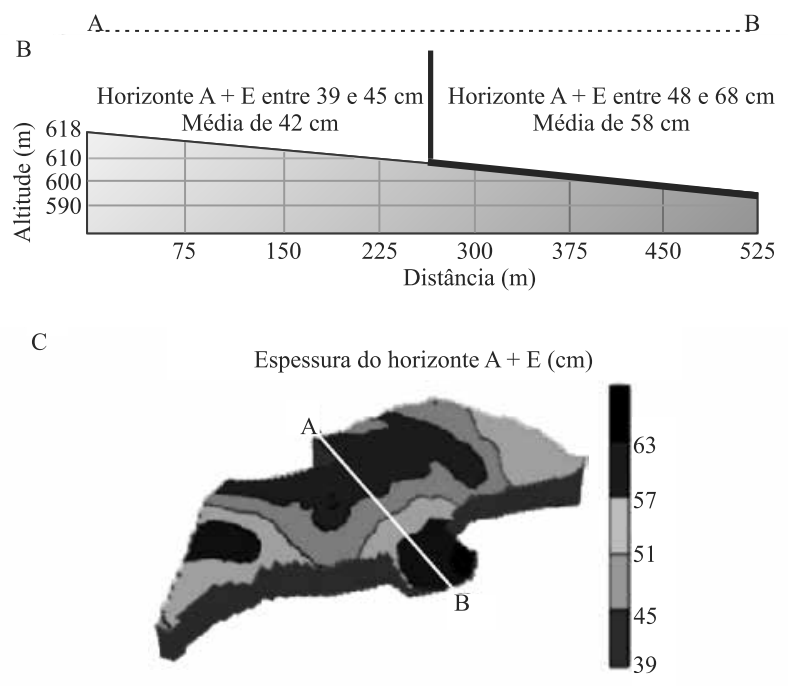

Figura 1. Localização da área e malha amostral (A); corte para representação do perfil de elevação e espessura do horizonte $\mathrm{A}+\mathrm{E}(\mathrm{B})$; modelo digital de elevação que representa a variabilidade espacial da espessura do horizonte $\mathrm{A}+\mathrm{E}$ (C). 
Consideraram-se para a análise os grãos retidos na peneira com abertura $17 \mathrm{~mm}$.

Os resultados das análises foliares do cafeeiro foram submetidos à metodologia do sistema integrado de diagnose e recomendação (DRIS). Esta técnica compara o balanço nutricional dos nutrientes na folha ao padrão nutricional ótimo, baseado em altos índices de produtividade (Beaufils, 1973). Para o cálculo pelo DRIS, utilizaram-se informações sobre os teores de N, $\mathrm{P}, \mathrm{K}, \mathrm{Ca}, \mathrm{Mg}, \mathrm{S}, \mathrm{B}, \mathrm{Cu}, \mathrm{Fe}, \mathrm{Mn}$ e $\mathrm{Zn}$, avaliados nas folhas de 1.557 plantas da unidade amostral.

Calcularam-se a média, o desvio-padrão (DP) e o coeficiente de variação $(\mathrm{CV})$ para as variáveis do solo e da planta. As médias das variáveis do solo e da planta, em relação à espessura do horizonte $\mathrm{A}+\mathrm{E}$, foram comparadas pelo teste de Tukey, a 5\% de probabilidade.

A dependência espacial foi avaliada pelo variograma simples e por mapas de krigagem (Isaaks \& Srivastava, 1989). A correlação espacial entre os parâmetros da cor e as variáveis da planta de café foi avaliada pelo variograma cruzado, que é ajustado somente para as variáveis que apresentam correlação entre si (Deutsch \& Journel, 1998). A técnica do variograma cruzado foi utilizada para avaliar a semelhança dos modelos matemáticos (variogramas) que descrevem a variabilidade espacial das variáveis do solo e da cultura. A dependência espacial entre as variáveis foi estimado pela equação 2 (Deutsch \& Journel, 1998), a seguir:

$$
\widehat{\gamma}_{z y}(h)=\frac{1}{2 N(h)} \sum_{i \neq}^{N(h)}\left[z\left(x_{i}\right)-z\left(x_{i}+h\right)\right]\left[y\left(x_{i}\right)-y\left(x_{i}+h\right)\right],
$$

em que: $\hat{\gamma}_{z y}(h)$ é a semivariância experimental cruzada, para uma distância de separação $\mathrm{h} ; \mathrm{z}\left(\mathrm{x}_{\mathrm{i}}\right)$ é o valor da variável principal (aquela a ser estimada), no ponto i; $\mathrm{y}\left(\mathrm{x}_{\mathrm{i}}\right)$ é o valor da variável secundária no ponto i; e $\mathrm{N}(\mathrm{h})$ é o número de pares de pontos separados pela distância h.

O variograma simples é um caso particular do variograma cruzado, em que a semivariância é calculada somente para uma propriedade e, assim, é considerado como uma medida da autocorrelação espacial da variável.

Os dados referentes aos 173 pontos de amostragem de solo foram hierarquizados em grupos, com base no percentil da produtividade e do parâmetro qualitativo percentagem de grãos retidos na peneira de $17 \mathrm{~mm}$ (Tabela 1).
A média dos resultados referentes ao valor, croma, matiz e IAV, para cada um dos grupos de resposta da planta, foi utilizada para construir gráficos de dispersão e representar a relação das variáveis dependentes (resposta da planta), em função das variáveis independentes (atributos da cor). Utilizouse o método dos mínimos quadrados, para ajustar o modelo de regressão ao conjunto de pontos das variáveis dependentes e independentes.

Construiu-se o triângulo de cor para identificar locais com diferentes potenciais de produtividade, com base no conceito do diagrama de cromaticidade elaborado a partir dos valores triestímulos (XYZ). A construção do triângulo foi feita em duas etapas, conforme a seguir. Na primeira etapa, os intervalos dos modelos de regressão para valor $(4,40$ a 4,67), croma $(5,00$ a 5,28$)$ e matiz $(6,99$ a 7,68$)$ foram subdivididos em quatro grupos, com base no intervalo do modelo de regressão da produtividade - 33 a 20 sacas ha $^{-1}, 20$ a 13 sacas ha-1 13 a 7 sacas ha $^{-1}$ e 7 a 3 sacas ha-1 $^{-1}$ (Figura 2).

$\mathrm{Na}$ segunda etapa, foi feita a integração dos triângulos para valor, croma e matiz. Cada intersecção das componentes da cor corresponde aos locais com diferentes características físicas e químicas que, por sua vez, condicionam diferentes potenciais de produtividade de café na área estudada. Esse tipo de representação gráfica elaborada a partir da ERD pode mostrar a interação existente entre os três parâmetros da cor, no sistema Munsell, e uma quarta variável de interesse agronômico.Assim, esta é uma alternativa rápida e viável, para a elaboração de triângulos de cor

Tabela 1. Valores médios dos atributos da cor resultantes dos grupos de resposta da planta.

\begin{tabular}{|c|c|c|c|c|}
\hline Grupo & Valor & Croma & Matiz & IAV \\
\hline & \multicolumn{4}{|c|}{ Produtividade } \\
\hline 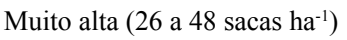 & 4,40 & 5,20 & 6,99 & 3,58 \\
\hline Alta $\left(16\right.$ a 25 sacas ha $\left.^{-1}\right)$ & 4,46 & 5,18 & 7,06 & 3,45 \\
\hline Média (10 a 15 sacas ha $\left.^{-1}\right)$ & 4,46 & 5,27 & 7,13 & 3,43 \\
\hline Baixa (6 a 9 sacas ha-1) & 4,51 & 5,08 & 7,27 & 3,12 \\
\hline \multirow[t]{2}{*}{ Muito baixa (0 a 5 sacas ha $\left.^{-1}\right)$} & 4,67 & 5,00 & 7,68 & 2,55 \\
\hline & \multicolumn{4}{|c|}{$\begin{array}{l}\text { Percentagem de grãos retidos na } \\
\text { peneira de } 17 \mathrm{~mm}\end{array}$} \\
\hline Muito alta (41 a 50\%) & 4,55 & 5,13 & 7,32 & 3,07 \\
\hline Alta $(37$ a $40 \%)$ & 4,57 & 5,07 & 7,41 & 2,93 \\
\hline Média (32 a 36\%) & 4,54 & 5,20 & 7,23 & 3,24 \\
\hline Baixa (29 a 31\%) & 4,46 & 5,19 & 7,09 & 3,43 \\
\hline Muito baixa (0 a $28 \%)$. & 4,44 & 5,15 & 7,15 & 3,35 \\
\hline
\end{tabular}

IAV: índice de avermelhamento. 
para diferentes regiões, com o objetivo de auxiliar a identificação de áreas de manejo no campo.

\section{Resultados e Discussão}

Os locais com menor espessura do horizonte $\mathrm{A}+\mathrm{E}$ (entre 39 e $45 \mathrm{~cm}$ ) apresentaram os maiores valores de DRIS $(8,28), \operatorname{IAV}(3,62)$ e produtividade $\left(26,13 \mathrm{sc} \mathrm{ha}^{-1}\right)$, e os menores valores para matiz $(6,94)$, valor $(4,33)$, croma $(5,10)$, percentagem de grãos retidos na peneira de $17 \mathrm{~mm}(31,33 \%)$, argila $(15,66 \%)$, carbono orgânico $\left(28,44 \mathrm{~g} \mathrm{dm}^{-3}\right)$, fósforo $\left(49,04 \mathrm{mg} \mathrm{dm}^{-3}\right)$ e soma de bases $\left(46,52 \mathrm{mmol}_{\mathrm{c}} \mathrm{dm}^{-3}\right)$. O maior valor do $\operatorname{IAV}(3,62)$, nos locais com menor espessura dos horizontes $\mathrm{A}+\mathrm{E}$, está relacionado ao maior teor de argila nessa camada, o que indica que estes solos foram relativamente mais erodidos e, assim, estão mais próximos do horizonte $\mathrm{B}$ textural, que é mais argiloso.

Com exceção do croma, a média de todas as outras variáveis relacionadas à cor do solo e à resposta da planta diferiram em relação às áreas e à espessura dos horizontes A+E. Observou-se maior desbalanço nutricional na parte superior da área de estudo, com maior IAV e menor espessura do horizonte A+E; nesse local, o valor de DRIS está mais distante de zero $(8,28)$ do que na parte inferior da área de estudo $(6,88)$, o que indica que parte dos nutrientes foi exportada para o desenvolvimento do grão. A maior produtividade na parte superior da área $\left(26,13 \mathrm{sc} \mathrm{ha}^{-1}\right)$, em comparação à parte inferior $\left(13,76 \mathrm{sc} \mathrm{ha}^{-1}\right)$, está de acordo com essa explicação.

A maioria das raízes do café, com maior potencial de absorção (menos de $3 \mathrm{~mm}$ de diâmetro), está localizada sob o dossel das plantas em até $0,20 \mathrm{~m}$ de profundidade (Motta et al., 2006). Mediante os resultados expostos, os locais na área com solo mais avermelhado (maior IAV)

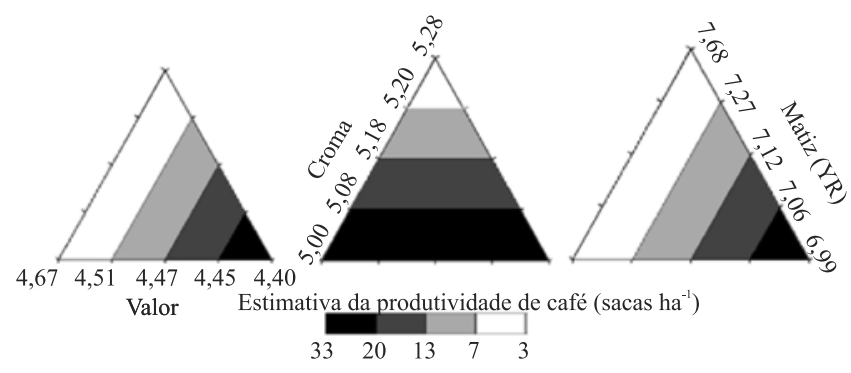

Figura 2. Triângulos de cor que relacionam a amplitude encontrada nos modelos de regressão da produtividade com o valor, o croma e o matiz. indicam menor espessura do horizonte $\mathrm{A}+\mathrm{E}$, ambiente onde há melhor absorção de água e nutrientes, o que aumenta o potencial produtivo. Nos locais em que a espessura do horizonte $\mathrm{A}+\mathrm{E}$ é maior ocorre maior percentagem de grãos retidos na peneira de $17 \mathrm{~mm}$, o que pode ser atribuído aos resultados da soma de bases (57,58 $\left.\mathrm{mmol}_{\mathrm{c}} \mathrm{dm}^{-3}\right)$, carbono orgânico $\left(40,22 \mathrm{~g} \mathrm{dm}^{-3}\right)$ e fósforo $\left(69,35 \mathrm{mg} \mathrm{dm}^{-3}\right)$, que confirmam a maior fertilidade do solo nesses locais.

Os locais menos avermelhados (maior espessura do horizonte $\mathrm{A}+\mathrm{E}$ ) apresentaram os maiores valores de CV dos atributos estudados, com exceção do valor, percentagem de grãos retidos na peneira de $17 \mathrm{~mm}$, fósforo e carbono orgânico. Sanchez et al. (2013) também relatam melhores ambientes de produção para a cultura do café, em locais com menor espessura do horizonte $\mathrm{A}+\mathrm{E}$.

O modelo esférico do variograma foi o que melhor se ajustou aos atributos matiz, croma, IAV, produtividade, DRIS e argila, enquanto o modelo exponencial foi o que melhor se ajustou aos atributos valor, percentagem de grãos retidos na peneira de $17 \mathrm{~mm}$, fósforo, carbono orgânico e soma de bases (Figura 3). O modelo esférico indica mudanças bruscas do padrão de variabilidade entre pontos mais distantes (Cambardella et al., 1994); o exponencial indica padrões de variabilidade mais irregulares em distâncias curtas (Isaaks \& Srivastava, 1989).

Os valores de alcance variaram de 150 a $479 \mathrm{~m}$, para os componentes da cor do solo, e de 150 a $180 \mathrm{~m}$ para a resposta da planta. Autores que realizaram estudos quanto à variabilidade espacial, em Argissolo originado de arenito do Planalto Ocidental Paulista, também encontraram valores de alcance dentro da mesma faixa de variação - 150 a $479 \mathrm{~m}$-, em área de citros (284 a $350 \mathrm{~m}$ ) (Siqueira et al., 2010) e canade-açúcar (160 a 410 m) (Sanchez et al., 2009). Essa similaridade do padrão de distribuição espacial pode estar associada à variabilidade inicial causada pelo material de origem (Schemiko et al., 2014; Fernandes \& Ribeiro, 2015) comum a estes trabalhos.

Observando-se os mapas de matiz, valor e croma, nota-se que os menores valores estão localizados na área com menor espessura do horizonte $\mathrm{A}+\mathrm{E}$ (Figura 1 B e C). Estes resultados são complementares aos da Tabela 2 e indicam os limites entre os ambientes com diferentes características e as classes de variabilidade. Produtividade e percentagem de grãos de café retidos 

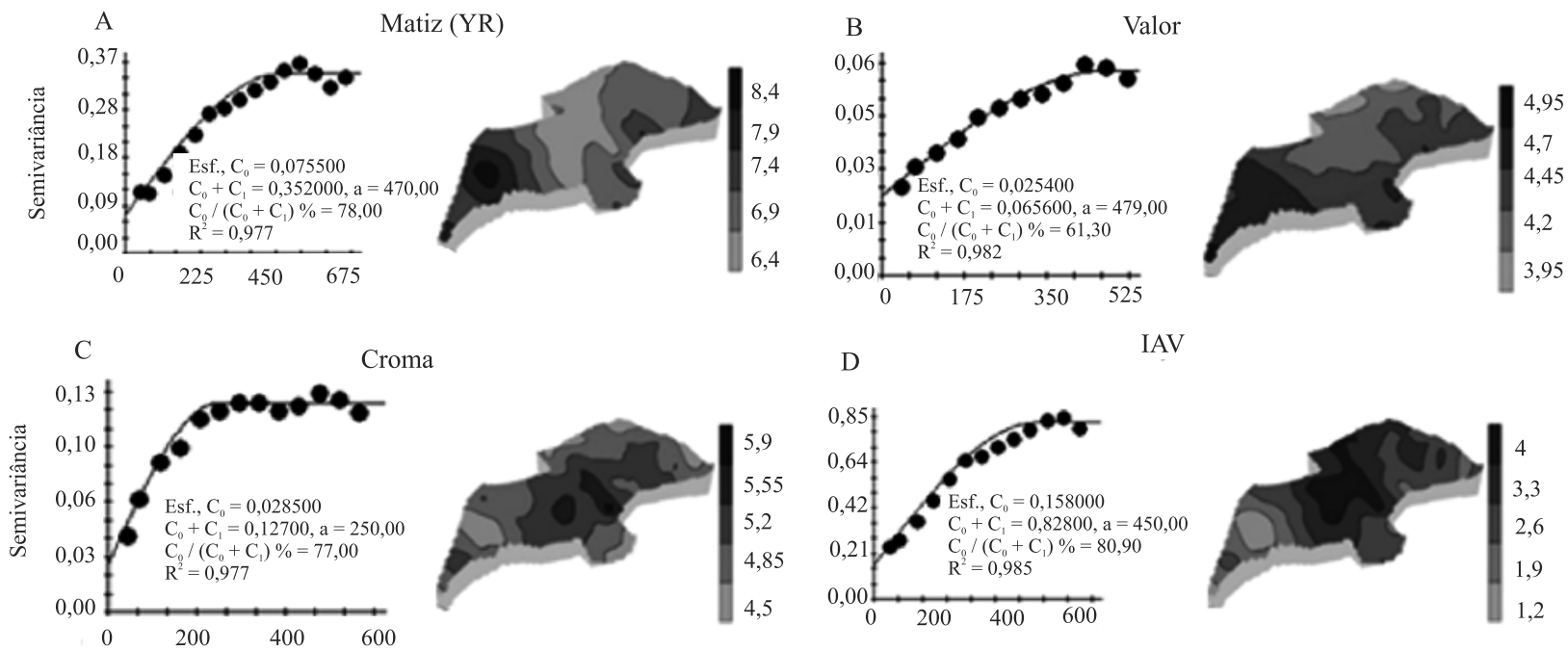

D

IAV
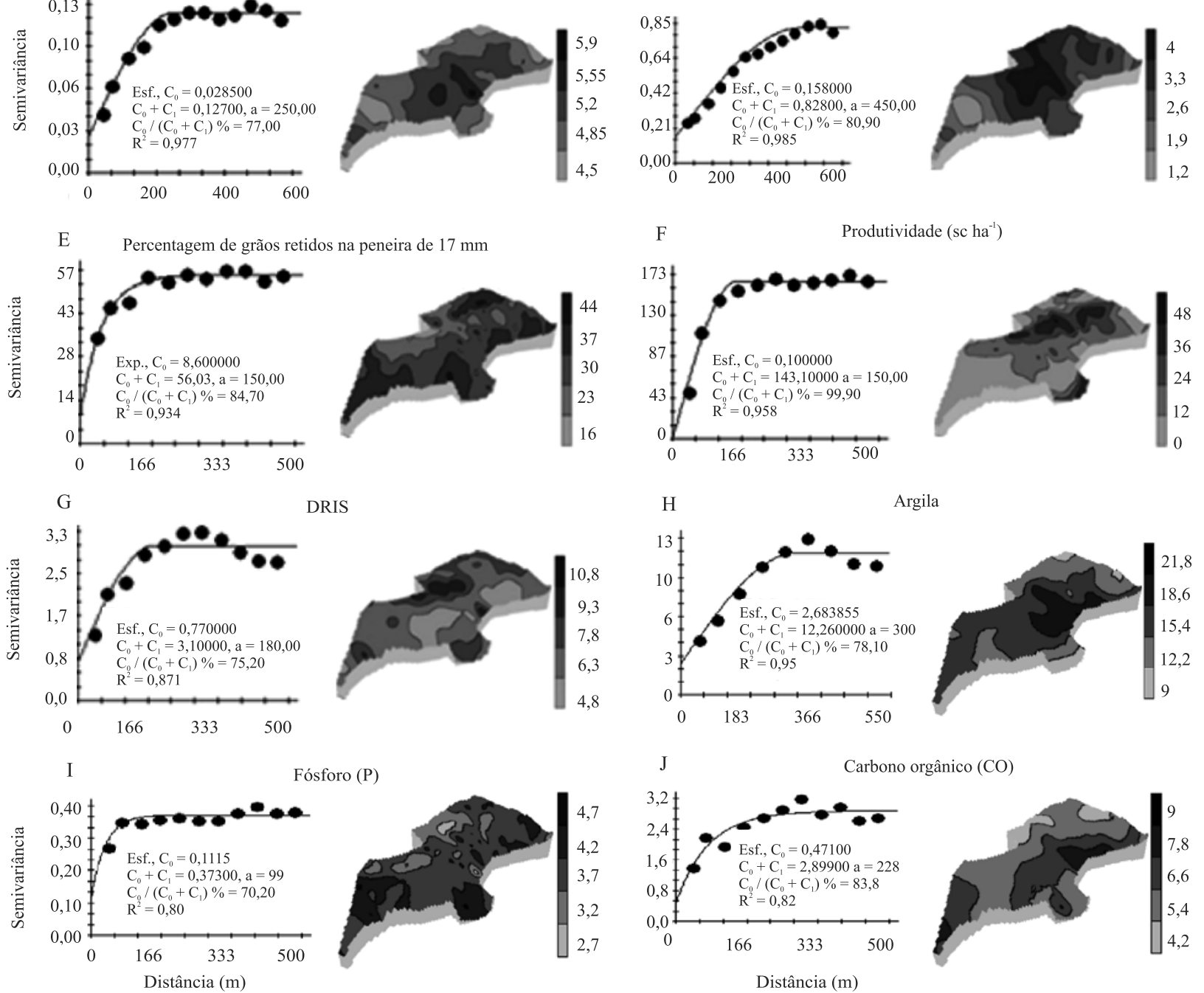

Fósforo (P)
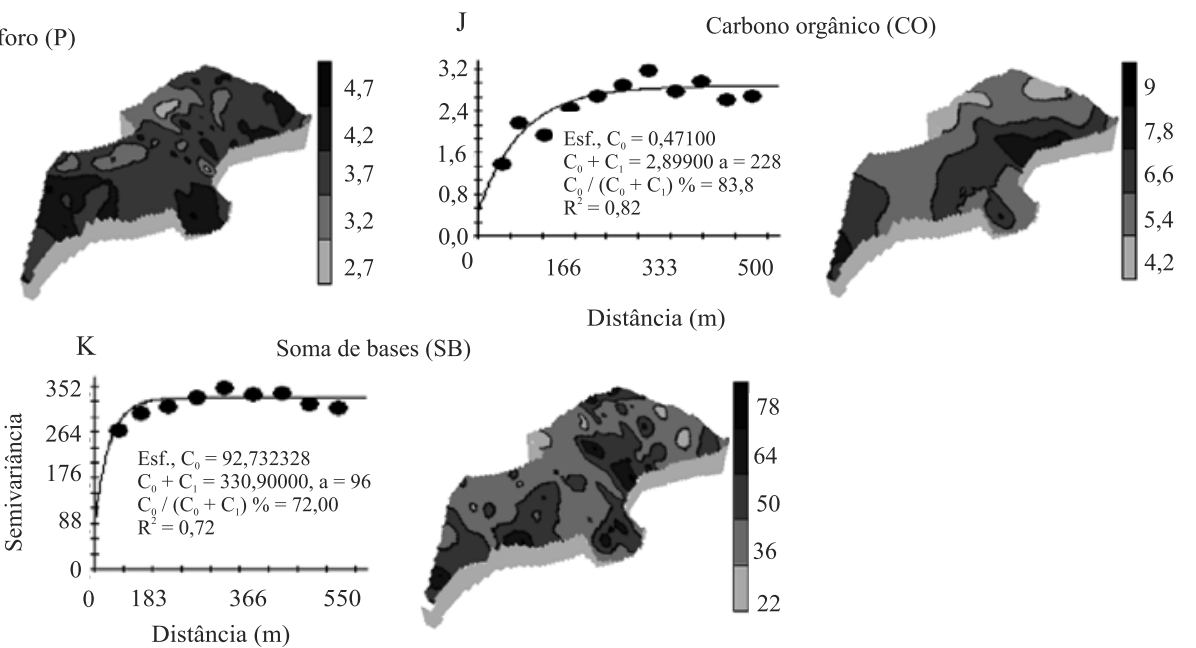

Figura 3. Variogramas e mapas de krigagem para matiz, valor, croma e índice de avermelhamento (IAV), à profundidade de 0,0-0,20 m, e percentagem de grãos retidos na peneira de $17 \mathrm{~mm}$, produtividade e DRIS. Esf., modelo esférico; Exp., modelo exponencial; Co, efeito pepita; $\mathrm{C}_{\mathrm{o}}+\mathrm{C}_{1}$, patamar; a, alcance; $\mathrm{C}_{0} /\left(\mathrm{C}_{0}+\mathrm{C}_{1}\right)$, grau de dependência espacial; $\mathrm{R}^{2}$, coeficiente de determinação. 
na peneira de $17 \mathrm{~mm}$ apresentaram padrão inverso de variabilidade. Os locais com menor espessura do horizonte $(39$ a $45 \mathrm{~cm}$ ) tiveram maior produtividade (36 a 60 sacas ha $^{-1}$ ) e de frutos menores (menos de 16\% dos frutos foram retidos na peneira de $17 \mathrm{~mm}$ ).

Observaram-se as seguintes relações de dependência positiva: do matiz em relação à percentagem de grãos retidos na peneira de $17 \mathrm{~mm}$ (449 m); do valor, à percentagem de grãos retidos na peneira de $17 \mathrm{~mm}$ (410 m); do croma, à produtividade $(207 \mathrm{~m})$; do IAV, à produtividade (259 m); e do IAV, à argila (473 m). Os variogramas cruzados dos pares - matizprodutividade, matiz-DRIS, IAV-percentagem de grãos retidos na peneira de $17 \mathrm{~mm}$, IAV-carbono orgânico, IAV-fósforo, IAV-soma de bases - apresentaram dependência espacial negativa (Figura 4). Estes resultados indicam que os componentes da cor obtidos pela ERD têm potencial para seu uso como covariáveis na cokrigagem e para auxiliar o detalhamento de mapas de produtividade e do tamanho do grão na cultura do café.

Modelos quadráticos foram encontrados para os atributos analisados. A produtividade pode ser estimada pelos componentes da cor do solo à profundidade de 0,0-0,2 m, com precisão de 66 a 95\% (Figura 5). Quanto às características do grão, os atributos da cor estimam o tamanho do grão com precisão de $88 \%$.
Para o DRIS, a precisão é de $82 \%$. Em solos originados de sedimentos do Grupo Barreiras, os componentes da cor do solo foram utilizados em relações diretas para estimar a fertilidade, com precisão de $85 \%$ (Resende, 2013); a erodibilidade foi estimada com precisão de 70 a 73\%; e a acurácia, com 53\% (Dantas et al., 2014b). Em solos com teor de $\mathrm{Fe}_{2} \mathrm{O}_{3}$ de 20 a $200 \mathrm{~g} \mathrm{~kg}^{-1}$, o IAV apresentou baixa precisão (4\%) na estimativa de áreas com diferentes potenciais de adsorção de fósforo (Peluco et al., 2015).

A representação gráfica com as três componentes da cor mostra que as áreas com maior potencial de

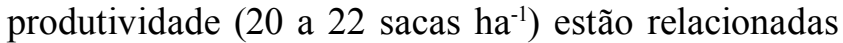
ao valor entre 4,45 e 4,40, matiz entre 7,09 e 6,99 e croma entre 5,0 e 5,08. Estas variações dos atributos da cor expressam indiretamente a variação do teor de argila entre 7 e $35 \%$, teores de fósforo entre 8 e 187 $\mathrm{mg} \mathrm{dm}{ }^{-3}$ e soma de bases entre 14 e $216 \mathrm{mmol}_{\mathrm{c}} \mathrm{dm}^{-3}$. Os locais com menor produtividade ( 3 a 7 sacas ha $^{-1}$ ) estão relacionados com o valor entre 4,51 e 4,67, matiz entre 7,27 e 7,68 e croma entre 5,20 e 5,28. Nestes locais, o teor de argila é de $16 \%$, os teores de fósforo variam entre 65 e $75 \mathrm{mg} \mathrm{dm}^{-3}$, e a soma de bases, entre 56 e 58 $\mathrm{mmol}_{\mathrm{c}} \mathrm{dm}^{-3}$ (Figura 6). A faixa espectral na região do visível - 370 a $750 \mathrm{~nm}$ - já é utilizada no detalhamento de áreas com diferentes potenciais produtivos para a cultura da cana-de-açúcar (Marques Jr. et al., 2014).

Tabela 2. Valores médios, desvio-padrão e coeficiente de variação (CV) dos componentes da cor do solo, à profundidade de 0,0-0,2 m, e características da cultura do café (produtividade e parâmetros qualitativos, $\mathrm{n}=173$ ) relacionados à espessura dos horizontes $\mathrm{A}+\mathrm{E}^{(1)}$.

\begin{tabular}{|c|c|c|c|c|c|c|}
\hline \multirow[t]{3}{*}{ Variável $^{(2)}$} & \multicolumn{6}{|c|}{ Espessura do horizonte $\mathrm{A}+\mathrm{E}^{(3)}$} \\
\hline & \multicolumn{2}{|c|}{ Média } & \multicolumn{2}{|c|}{ Desvio-padrão } & \multicolumn{2}{|c|}{ CV $(\%)$} \\
\hline & Menor & Maior & Menor & Maior & Menor & Maior \\
\hline Teor de argila & $15.66 b$ & $16.78 \mathrm{a}$ & 2.74 & 4.03 & 17.50 & 24.02 \\
\hline Fósforo & $49.04 b$ & $69.35 \mathrm{a}$ & 28.18 & 36.55 & 57.45 & 52.70 \\
\hline Carbono orgânico & $28.44 b$ & $40.22 \mathrm{a}$ & 16.40 & 21.18 & 57.57 & 52.65 \\
\hline Soma de bases & $46.52 b$ & $57.58 \mathrm{a}$ & 18.48 & 29.45 & 39.73 & 51.15 \\
\hline Matiz & $6.94 \mathrm{~b}$ & $7.43 \mathrm{a}$ & 0.34 & 0.58 & 4.9 & 7.81 \\
\hline Valor & $4.33 b$ & $4.62 \mathrm{a}$ & 0.23 & 0.23 & 5.31 & 4.98 \\
\hline Croma & $5.10 \mathrm{a}$ & $5.19 \mathrm{a}$ & 0.28 & 0.37 & 5.49 & 7.13 \\
\hline IAV & $3.62 \mathrm{a}$ & $2.94 b$ & 0.56 & 0.88 & 15.47 & 29.93 \\
\hline DRIS & $8.28 \mathrm{a}$ & $6.88 b$ & 1.61 & 1.53 & 19.44 & 22.24 \\
\hline Produtividade (sacas ha ${ }^{-1}$ ) & $26.13 \mathrm{a}$ & $13.76 b$ & 15.19 & 10.47 & 58.13 & 76.09 \\
\hline Peneira & $31.33 b$ & $35.8 \mathrm{a}$ & 8.08 & 5.99 & 25.79 & 16.73 \\
\hline
\end{tabular}

${ }^{(1)}$ Médias seguidas de letras iguais, nas linhas, não diferem entre si, pelo teste de Tukey, a 5\% de probabilidade. (2)Matiz, Valor e Croma - Componentes da cor do solo, obtidas por espectroscopia de reflectância difusa; IAV, índice de avermelhamento do solo; DRIS, índice de diagnose foliar; peneira, percentagem de grãos retidos na peneira de $17 \mathrm{~mm} .{ }^{(3)}$ Espessura do horizonte A+E: menor, entre 39 e $45 \mathrm{~cm}$; maior, entre 48 e $68 \mathrm{~cm}$. 

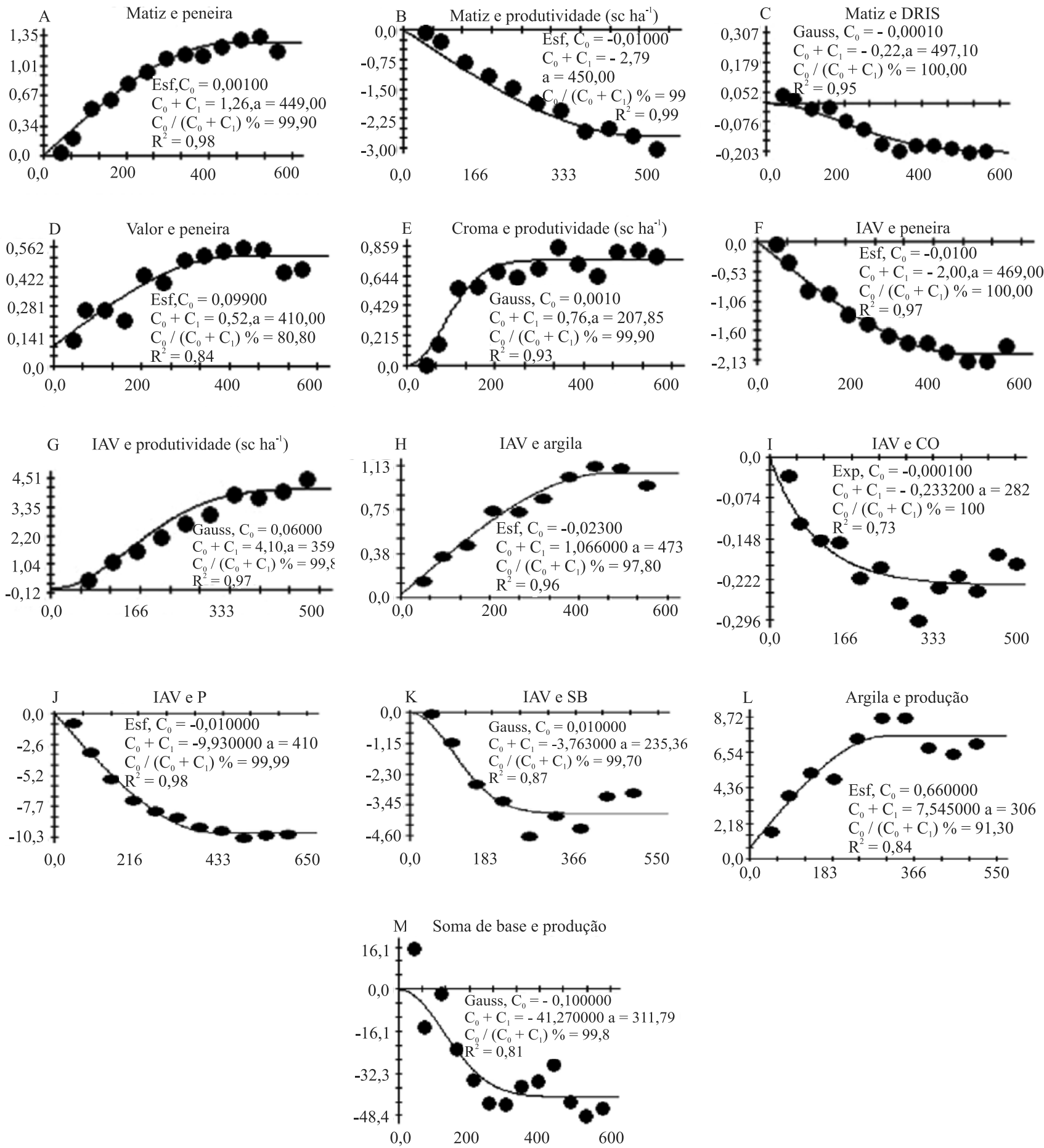

Figura 4. Variogramas cruzados dos atributos de cor, à profundidade de 0,0-0,20 m, produtividade, qualidade do grão de café e atributos físicos e químicos do solo. Esf., modelo esférico; Exp., modelo exponencial; Gauss, modelo gaussiano; Co, efeito pepita; $\mathrm{Co}+\mathrm{C}_{1}$ : patamar; a, alcance; $\mathrm{C}_{0} /\left(\mathrm{C}_{0}+\mathrm{C}_{1}\right)$, grau de dependência espacial; $\mathrm{R}^{2}$, coeficiente de determinação. 

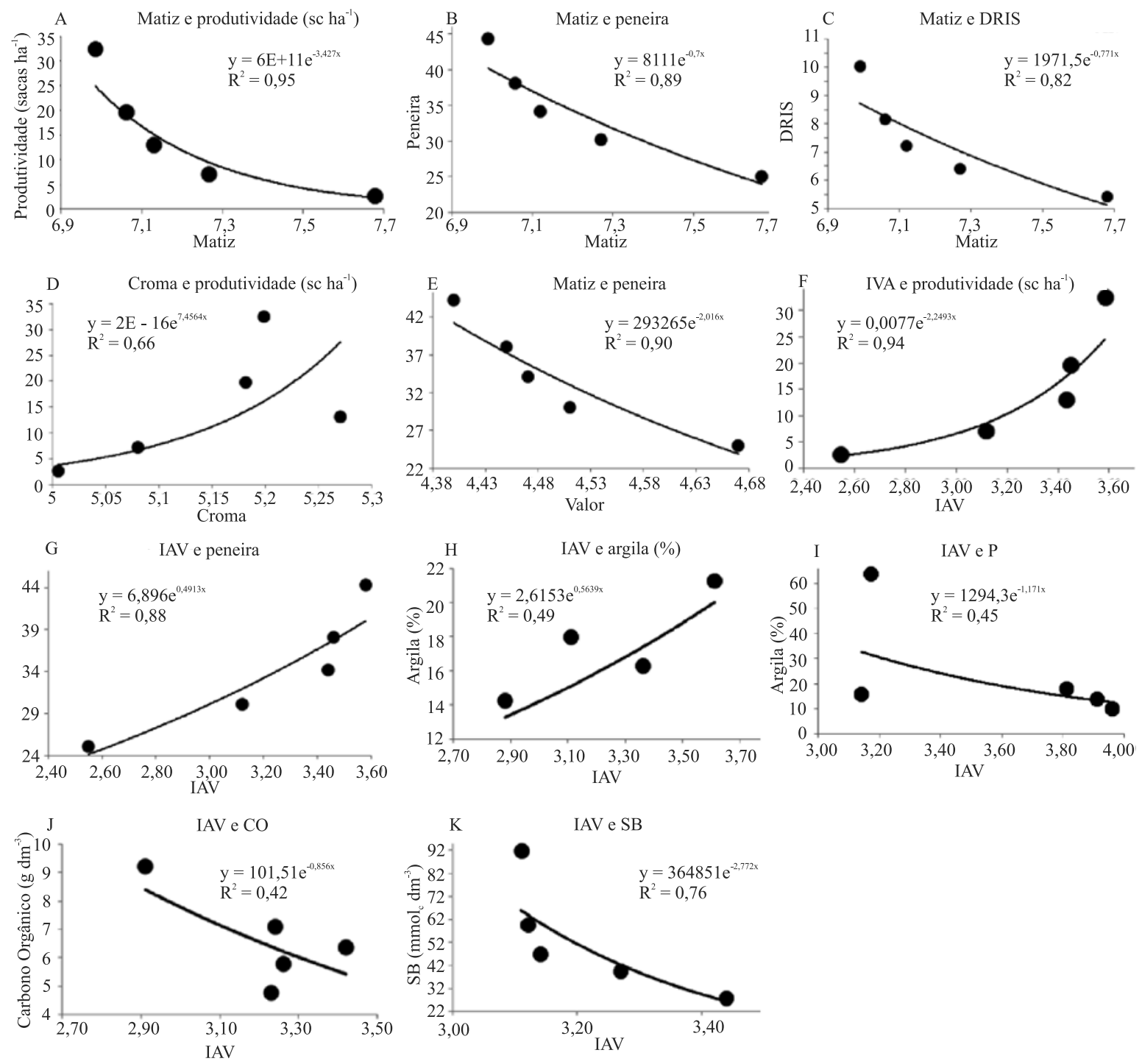

Figura 5. Modelos de regressão dos atributos da cor do solo, à profundidade de $0,0-0,20 \mathrm{~m}$, com a produtividade, qualidade do grão de café e atributos físicos e químicos do solo.

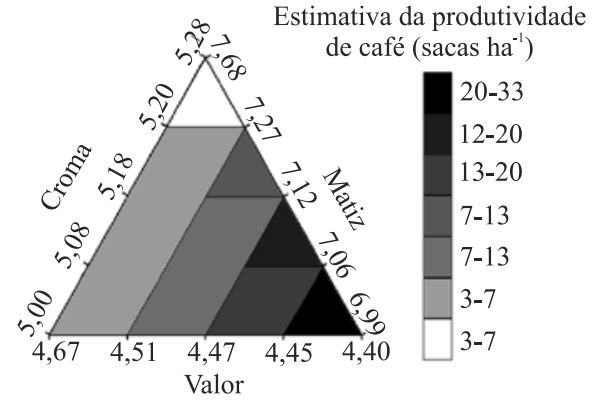

Figura 6. Produtividade do cafeeiro "Coffea arabica 'Catuaí Amarelo IAC 62' em diferentes ambientes de Argissolo Vermelho-Amarelo com baixo teor de ferro total caracterizados pelos componentes da cor obtida por ERD.

\section{Conclusões}

1. A cor do solo, obtida pela espectroscopia de reflectância difusa(ERD), é eficiente tanto na identificação de áreas com diferentes potenciais de produtividade quanto de qualidade do grão de café.

2. O método indireto de caracterização da cor pela ERD pode ser uma alternativa para viabilizar futuros trabalhos, que busquem identificar áreas quanto à produtividade e qualidade de grão de café em grandes áreas. 


\section{Agradecimentos}

À Fundação de Amparo à Pesquisa do Estado do São Paulo (Fapesp, processo no. 11/07549-2), pelo apoio financeiro; e ao Conselho Nacional de Desenvolvimento Científico e Tecnológico (CNPq), pela concessão de bolsa de produtividade.

\section{Referências}

BAHIA, A.S.R. de S.; MARQUES JR., J.; SIQUEIRA, D.S. Procedures using diffuse reflectance spectroscopy for estimating hematite and goethite in Oxisols of São Paulo, Brazil. Geoderma Regional, v.5, p.150-156, 2015. DOI: 10.1016/j.geodrs.2015.04.006.

BARRÓN, V.; MELLO, J. W. V.; TORRENT, J. Caracterização de óxidos de ferro em solos por espectroscopia de reflectância difusa. In: NOVAIS, R.F. de; ALVAREZ V., V.H.; SCHAEFER, C.E.G.R., (Ed.). Tópicos em ciência do solo. Viçosa: Sociedade Brasileira de Ciência do Solo, 2000. v.1, p.139-162.

BARRÓN, V.; TORRENT, J. Iron, manganese and aluminium oxides and oxyhydroxides. In: NIETO, F.; LIVI, K.J.T.; OBERTI, R. (Ed.). Minerals at the nanoscale. London: European Mineralogical Union: Mineralogical Society of Great Britain \& Ireland, 2013. p.297-336. (European Mineralogical Union Notes in Mineralogy, v.14). DOI: 10.1180/EMU-notes.14.9.

BEAUFILS, E.R. Diagnosis and recommendation integrated system (DRIS): a general scheme of experimentation and calibration based on principles developed from research in plant nutrition. Pietermaritzburg: University of Natal, 1973. 132p. (Soil Science Bulletin, 1).

CAMARGO, L.A.; MARQUES JÚNIOR., J.; BARRÓN, V.; ALLEONI, L.R.F.; BARBOSA, R.S.; PEREIRA, G.T. Mapping of clay, iron oxide and adsorbed phosphate in Oxisols using diffuse reflectance spectroscopy. Geoderma, v.251/252, p.124-132, 2015. DOI: 10.1016/j.geoderma.2015.03.027.

CAMBARDELLA, C.A.; MOORMAN, T.B.; NOVAK, J.M.; PARKIN, T.B.; KARLEN, D.L.; TURCO, R.F.; KONOPKA, A.E. Field-scale variability of soil properties in Central Iowa soils. Soil Science Society of America Journal, v.58, p.1501-1511, 1994. DOI: $10.2136 /$ sssaj1994.03615995005800050033x.

CAMPOS, R.C.; DEMATTÊ, J.A.M. Cor do solo: uma abordagem da forma convencional de obtenção em oposição à automatização do método para fins de classificação de solos. Revista Brasileira de Ciência do Solo, v.28, p.853-863, 2004. DOI: 10.1590/S010006832004000500008 .

CLAESSEN, M.E.C. (Org.). Manual de métodos de análise de solo. 2.ed. rev. e atual. Rio de Janeiro,: EMBRAPA-CNPS, 1997. $212 \mathrm{p}$.

DANTAS, J.S.; MARQUES JÚNIOR, J.; MARTINS FILHO, M.V.; RESENDE, J.M. do A.; CAMARGO, L.A.; BARBOSA, R.S. Gênese de solos coesos do leste maranhense: relação solopaisagem. Revista Brasileira de Ciência do Solo, v.38, p.10391050, 2014a. DOI: 10.1590/S0100-06832014000400001.
DANTAS, J.S.; MARTINS FILHO, M.V.; MARQUES JÚNIOR, J.; RESENDE, J.M. do A.; TEIXEIRA, D.D.B.; BARBOSA, R.S.; SIQUEIRA, D.S. Coeficiente de erodibilidade em sulcos e entressulcos de Argissolos coesos estimado pela cor do solo. Pesquisa Agropecuária Brasileira, v.49, p.700-707, 2014b. DOI: 10.1590/S0100-204X2014000900006.

DAY, P. R. Particle fraction and particle-size analysis. In: BLACK, C.A. (Ed.). Methods of soil analysis. Madison: American Society of Agronomy, 1965. v.1, p.545-566. (ASA. Agronomy, 9).

DEUTSCH, C.V.; JOURNEL, A.G. GSLIB: Geostatistical Software Library and User's Guide. 2.ed. Oxford: Oxford University Press, 1998. 366p.

FERNANDES, L.A.; RIBEIRO, C.M.M. Evolution and palaeoenvironment of the Bauru Basin (Upper Cretaceous, Brazil). Journal of South American Earth Sciences, v.61, p.7190, 2015. DOI: 10.1016/j.jsames.2014.11.007.

IPT. INSTITUTO DE PESQUISAS TECNOLÓGICAS DO ESTADO DE SÃO PAULO. Mapa geomorfológico do Estado de São Paulo: mapas. São Paulo, 1981. 94p. (IPT Publicação, 1183; IPT Monografia, 5).

ISAAKS, E.H.; SRIVASTAVA, R.M. An introduction to applied geoestatistics. New York: Oxford University Press, 1989. 560p.

MARQUES JR., J.; SIQUEIRA, D.S.; CAMARGO, L.A.; TEIXEIRA, D.D.B.; BARRÓN, V.; TORRENT, J. Magnetic susceptibility and diffuse reflectance spectroscopy to characterize the spatial variability of soil properties in a Brazilian Haplustalf. Geoderma, v.219/220, p.63-71, 2014. DOI: 10.1016/j. geoderma.2013.12.007.

MOTTA, A.C.V.; NICK, J.A.; YORINORI, G.T.; SERRAT, B.M. Distribuição horizontal e vertical da fertilidade do solo e das raízes de cafeeiro (Coffea arabica L.) cultivar Catuaí. Acta Scientiarum. Agronomy, v.28, p.455-463, 2006. DOI: 10.4025/ actasciagron.v28i4.758.

PELUCO, R.G.; MARQUES JÚNIOR, J.; SIQUEIRA, D.S.; PEREIRA, G.T; BARBOSA, R.S.; TEIXEIRA, D. de B. Mapeamento do fósforo adsorvido por meio da cor e da suscetibilidade magnética do solo. Pesquisa Agropecuária Brasileira, v.50, p.259-266, 2015. DOI: 10.1590/S0100204X2015000300010.

POLLO, G.Z. Suscetibilidade magnética, atributos do solo e da planta na discriminação de áreas de manejo específico na cultura do café. 2013. 58p. Dissertação (Mestrado) - Universidade Estadual Paulista, Jaboticabal.

RAIJ, B. van; QUAGGIO, J.A.; CANTARELLA, J.; FERREIRA, M.E.; LOPES, A.S.; BATAGLIA, O.C. Análise química do solo para fins de fertilidade. Campinas: Fundação Cargill, 1987. $170 \mathrm{p}$.

RESENDE, J.M. do A. Caracterização pedométrica de atributos de Argissolos coesos do leste maranhense. 2013. 83p. Tese (Doutorado) - Universidade Estadual Paulista, Jaboticabal.

RESENDE, J.M. do A.; MARQUES JÚNIOR, J.; MARTINS FILHO, M.V.; DANTAS, J.S.; SIQUEIRA, D.S.; TEIXEIRA, D.D.B. Variabilidade espacial de atributos de solos coesos do 
leste Maranhense. Revista Brasileira de Ciência do Solo, v.38, p.1077-1090, 2014.

SANCHEZ, M.G.B.; MARQUES JR., J.; SIQUEIRA, D.S.; CAMARGO, L.A.; PEREIRA, G.T. Delineation of specific management areas for coffee cultivation based on the soil-relief relationship and numerical classification. Precision Agriculture, v.14, p.201-214, 2013. DOI: 10.1007/s11119-012-9288-z.

SANCHEZ, R.B.; MARQUES JÚNIOR, J.; SOUZA, Z.M. de; PEREIRA, G.T.; MARTINS FILHO, M.V. Variabilidade espacial de atributos do solo e de fatores de erosão em diferentes pedoformas. Bragantia, v.68, p.1095-1103, 2009. DOI: 10.1590/ S0006-87052009000400030.

SANTOS, H.G. dos; JACOMINE, P.K.T.; ANJOS, L.H.C. dos; OLIVEIRA, V.A. de; OLIVEIRA, J.B. de; COELHO, M.R.; LUMBRERAS, J.F.; CUNHA, T.J.F. (Ed.). Sistema brasileiro de classificação de solos. 2.ed. Brasília: Embrapa, 2006. 306p.

SCHEMIKO, D.C.B.; VESELY, F.F.; FERNANDES, L.A.; SOWEK, G.A. Distinção dos elementos deposicionais fluviais, eólicos e lacustres do Membro Morro Pelado, Permiano Superior da Bacia do Paraná. Geologia USP. Série Científica, v.14, p.2946, 2014. DOI: 10.5327/Z1519-874X201400030003.

SIQUEIRA, D.S.; MARQUES JR., J.; PEREIRA, G.T. The use of landforms to predict the variability of soil and orange attributes. Geoderma, v.155, p.55-66, 2010. DOI: 10.1016/j. geoderma.2009.11.024.

THORNTHWAITE, C.W. An approach toward a rational classification of climate. Geographical Review, v.38, p.55-94, 1948. DOI: $10.2307 / 210739$.

TORRENT, J.; BARRÓN, V. Diffuse reflectance spectroscopy. In: ULERY, A.L.; DREES, L.R. (Ed.). Methods of soil analysis: part 5: mineralogical methods. Madison: Soil Science Society of America, 2008. p.367-387. (SSSA Book Series, 5). DOI: 10.2136/ sssabookser5.5.c13.

VISCARRA ROSSEL, R.A., BUI, E.N.; CARITAT, P. de; MCKENZIE, N.J. Mapping iron oxides and the color of Australian soil using visible-near-infrared reflectance spectra. Journal of Geophysical Research, v.115, p.1-13, 2010. DOI: 10.1029/2009JF001645.

WYSZECKI, G.; STILES, W.S. Color science: concepts and methods, quantitative data and formulae. 2.ed. New York: John Wiley \& Sons, 1982. 968p.

XIE, H.T.; YANG, X.M.; DRURY, C.F.; YANG, J.Y.; ZHANG, X.D. Predicting soil organic carbon and total nitrogen using mid- and near-infrared spectra for Brookston clay loam soil in Southwestern Ontario, Canada. Canadian Journal of Soil Science, v.91, p.53-63, 2011. DOI: 10.4141/cjss10029.

Recebido em 2 de junho de 2015 e aprovado em 24 de novembro de 2016 